\title{
Image Segmentation for Uneven Lighting Images based on Initial Window Size Selection
}

\author{
Rashmi Saini \\ Assistant Professor, \\ Govind Ballabh Pant \\ Enginering College, \\ Pauri Garhwal \\ Uttarakhand
}

\begin{abstract}
Thresholding is a simple but effective technique for image segmentation. In this paper, a novel method is presented to address the problem of image segmentation for uneven lighting images that is based on dynamic size of window. In pyramid approach (window merging), segmentation accuracy depends on the initial size selection. The proposed method is based on the concept of window growing approach, in which after selecting the initial window, selection criterion is tested. If a sub-image or window does not satisfies the selection criterion, instead of merging with neighboring window (pyramid approach), window is incremented by small value. This process is repeated until it satisfies the given selection criterion. Thereafter segment the window by thresholding method. In the proposed method, initial size of window is computed at run time, which is based on image statistics. This method provides superior image segmentation over existing thresholding methods for images that are degraded, uneven illumination and suffer from the problem like shadow.
\end{abstract}

\section{General Terms}

Image segmentation; thresholding; window based;

\section{Keywords}

initial size selection; sub-image.

\section{INTRODUCTION}

Thresholding is considered one of the popular and effective technique for image segmentation. In this paper, the proposed method is concerned with bi-level thresholding, which is also referred to as image binarization. A thresholding algorithm can compute either a single global threshold for the entire image or local thresholds applicable to sub-regions or windows of an image, which are adaptive to local image characteristics [14]. Sezgin and Sankur [11] categorize the thresholding methods in six groups according to the information they are exploiting: histogram shape, clustering of gray-level values, image entropy, general image attributes, spatial information, and local characteristics. A good adaptive thresholding algorithm produce the same segmentation result for uneven lighting image as a global thresholding algorithm produce when applied to a perfectly evenly lighting image [9]. Image thresholding is still a relevant research field and a number of method has been proposed in literature to address the problem of uneven lighting condition [2,5,6,7,9]. Generally, local thresholding methods are considered superior to the global ones for uneven illumination condition. Huang[2] proposed a method By adaptively selecting image window size based on the pyramid data structure manipulation of Lorentz information Measure(LIM). Although, the method produces good result but segmentation accuracy depend on the initial size of window. Sauvola[8] method compute a threshold value for each pixel that is based on image statistics, but in images where the gray values of foreground and background pixels are close to each other, the results degrade significantly. Pierre D. Wellner[9] presented a method, that is based on the scan line approach and calculating a moving average value of the last $n$ number of pixels seen. When the value of a pixel is significantly lower than this average value it is set to black, otherwise it is left white. Only one pass through the image is necessary, and the beauty of this algorithm is its simplicity but the drawback of this algorithm is that the segmentation result depends on the order of scanning. Darek bradley [6] presented a simple and efficient method using the integral image. This technique is an extension to a previous method[Wellner 1993], increase robustness to strong illumination changes. Because this method need to perform two iterations through each image instead of just one as in Wellner's method[9], due to this, perform slower. In this paper, a novel method of thresholding in partitioned windows is proposed. The technique is based on the window growing approach, and the window size is adaptively selected according entropy based selection criterion. The advantages of the technique are disturbance of uneven lighting is effectively eliminated after segmentation in partitioned window.

\section{Otsu's METHOD}

Let the pixels of a given picture be represented in L gray levels $[1,2,, \mathrm{~L}]$. The number of pixels at level $\mathrm{i}$ is denoted by $\mathrm{ni}$ and the total number of pixels by $\mathrm{N}=\mathrm{n} 1+\mathrm{n} 2+\ldots+\mathrm{nL} * \mathrm{In}$ order to simplify the discussion, the gray-level histogram is normalized and regarded as a probability distribution:

$$
p_{i}=\frac{n_{i}}{N}, \quad p_{i} \geq 0, \sum_{i=1}^{L} p_{i}=1
$$

Now suppose that dichotomize the pixels into two classes $C_{0}$ and $C_{I}$ (background and objects, or vice versa) by a threshold at level k; $C_{0}$ denotes pixels with levels $[1, k]$, and $C_{l}$ denotes pixels with levels $[k+1, L]$. Then the probabilities of class occurrence and the class mean levels, respectively, are given by

$$
\begin{gathered}
w_{o}=\operatorname{Pr}\left(C_{o}\right)=\sum_{i=1}^{k} p_{i}=w(k) \\
w_{1}=\operatorname{Pr}\left(C_{1}\right)=\sum_{i=k+1}^{L} p_{i}=1-w(k)
\end{gathered}
$$

And

$$
\mu_{o}=\sum_{i=1}^{k} i p_{i} / w_{o}=\mu(k) / w(k)
$$




$$
\mu_{1}=\frac{\sum_{i=k+1}^{L} i p_{i}}{w_{1}}=\frac{\mu_{T}-\mu(k)}{1-w(k)}
$$

Where

$$
\begin{array}{r}
w(k)=\sum_{i=1}^{k} p_{i}, \text { ? }(k)=\sum_{i=1}^{k} i p_{i},(6) \\
{ }_{T}=\quad \text { ? }(L)=\sum_{i=1}^{L} i p_{i}
\end{array}
$$

is the total mean level of the original picture. This can easily verify the following relation for any choice of $\mathrm{k}$ :

$$
w_{o} \text { ? }_{o}+w_{1} ?_{1}=?_{T}, \quad w_{o}+w_{1}=1
$$

The class variance is given by

$$
\begin{array}{r}
\sigma_{o}^{2}=\sum_{i=1}^{k}\left(i-\mu_{o}\right)^{2} p_{i} / w_{o} \\
\sigma_{1}^{2}=\sum_{i=k+1}^{L}\left(i-\mu_{1}\right)^{2} p_{i} / w_{1}
\end{array}
$$

In order to evaluate the "goodness" of the threshold (at level $k$ ), introduce the following discriminant criterion measures (or measures of class separability) used in the discriminant analysis:

$$
\lambda=\frac{\sigma_{B}^{2}}{\sigma_{W}^{2}}, \quad K=\frac{\sigma_{T}^{2}}{\sigma_{W}^{2}}, \eta=\frac{\sigma_{B}^{2}}{\sigma_{T}^{2}}
$$

Where

$$
\begin{aligned}
& \sigma_{W}^{2}=w_{o} \sigma_{0}^{2}+w_{1} \sigma_{1}^{2}, \sigma_{B}^{2}=w_{o}\left(\text { ? }_{o}-{ }_{T}\right)^{2}+ \\
& w_{1}\left(\text { (日) }_{1}-{ }_{T}\right)^{2}=w_{0} w_{1}\left(a_{0}-?_{1}\right)^{2} \\
& \sigma_{T}^{2}=\sum_{i=1}^{L}\left(i-\vartheta_{T}\right)^{2} p_{i}
\end{aligned}
$$

are the within-class variance, the between-class variance, and the total variance of levels, respectively. It is noticed that $\sigma_{W}^{2}$ and $\sigma_{B}^{2}$ are functions of threshold level $\mathrm{k}$, but $\sigma_{T}^{2}$ is independent of $\mathrm{k}$. Therefore, $\eta$ is the simplest measure with respect to $\mathrm{k}$. Thus adopt $\eta$ as the criterion measure to evaluate the "goodness" (or separability) of the threshold at level $\mathrm{k}$. The optimal threshold $\mathrm{k}^{*}$ that maximizes $\eta$

$$
\begin{gathered}
\eta(k)=\sigma_{B}^{2}(k) / \sigma_{T}^{2}, \\
\sigma_{B}^{2}(k)=\frac{\left[\square_{T} w(k)-\square(k)\right]^{2}}{w(k)[1-w(k)]}
\end{gathered}
$$

and the optimal threshold $\mathrm{k}^{*}$ is

$$
\sigma_{B}^{2}\left(k^{*}\right)=\max \left(\sigma_{B}^{2}(k)\right) 1 \leq k<L \quad(15)
$$

\section{PROPOSED METHOD}

The proposed work is based on two steps: a method to partition the image into dynamic windows by using incremental windowing technique followed by a thresholding technique on those windows. In the proposed work, problem of uneven illumination condition has been addressed using adaptive windowing technique, incremental window based selection and selected window is segmented by using Otsu's method[1] to improve the segmentation result.

\subsection{Initial Size Selction}

When a window or sub-image is tested against the selection criterion and if it satisfies the desired condition then window is fixed to be segment. Thereafter program requires new window to test the selection criterion, ideally the new window size should be one and every time when it fail to met the desired condition it must be enhance by a unit value. Practically the following procedure will increase the complexity to the algorithm. While in window merging approach segmentation accuracy greatly depends on the proper choice of initial window size. In order to overcome the above limitations, we proposed a method to determine the optimal initial window size that is based on the image statistics and computed at run time for the given image. In the proposed method every time when desired condition does not met window size should be incremented by $\Delta w$, in our method we kept this value one to get more accurate segmentation. Size of window is inversely proportional to the number of gray level in the image (for 8-bit image this value is 256). The entropy is one measure of information content of an image. The entropy of a given image has a minimum value of zero when all pixels have same intensity. So we conclude that if entropy is higher, the window size must be smaller. Variance is a measure of the contrast in digital images and standard deviation is the square root of the variance. Size of image is also considered to compute initial size of window. Initial size is calculated from the following formula.

$$
\text { Int }_{\text {size }}=\left\lceil\frac{K * \text { std } \times \max (\text { height,width })}{\text { Entropy } \times G L}\right\rceil
$$

Where std is the standard deviation of image, height and width are dimensions of the image, Entropy is the entropy of the whole image, GL is the No. gray level for the image .Here all images are 8-bit bit so $G L$ is 256. In order to control the initial size constant $\mathrm{K}$ is used and its value is fixed 2.0 by using error and trial approach. Entropy of each window $\mathrm{H}_{\mathrm{W}}$ is computed and is compared with the entropy of the total image. Entropy of gray level image is defined as

$$
H=-\sum_{i=1}^{256} p_{i} \ln p_{i}
$$

where $p_{i}$ is the probability of the $i^{\text {th }}$ gray value. Analogously, the window entropy is defined as

$$
H_{w}=-\sum_{i=1}^{256} p_{w i} \ln p_{w i}
$$

Where $p_{w i}$ is the probability of the $i^{\text {th }}$ gray value within the window.A window or sub-image is selected for segmentation if it satisfies the given selection criterion. If the current window doesn't fulfill the selected criterion then it is merged with the neighboring windows to form the next level of window

$$
\mathrm{H}_{\boldsymbol{w}} \geq \mathrm{CH}
$$

Where $C$ is a positive real constant. In our case $\mathrm{C}$ varies from $0-1$. The notion of fixing $\mathrm{C}$ between $0-1$ is to intuitively expect that the maximum entropy of the window could be the entropy of the whole image. Fig. 1 shows the algorithm in brief.

\subsection{Proposed algorithm}

Start

1) Compute initial size of window using equation (1) from proposed method.

2) Compute entropy of the whole image by using equation number (2). 
3) Compute entropy of the selected window using equation number (3).

4) Check out the selection criteria using equation number (4).

5) If current window satisfies the required condition then fix the window size and segment the current window by thresholding(otsu's method).

6) Else increment the size of window by incremental value $\Delta \mathrm{w}$ and repeat the steps from step 4 to step 6 .

7) Stop if whole image is segmented.

\subsection{Result Evaluation}

To evaluate the performance of proposed method we have used performance matrix misclassification error. The quality of segmentation result may be quantitatively evaluated by using Misclassification Error (ME) measure [20]. The measure reflects the percentage of background pixels erroneously classified into foreground, and conversely, foreground pixel erroneously assigned to background. For a two class segmentation problem, $M E$ can be simply formulated as

$$
M E=1-\frac{\left|B_{o} \cap B_{T}\right|+\left|F_{o} \cap F_{T}\right|}{\left|B_{0}\right|+\left|F_{0}\right|}
$$

Where $B_{o}$ and $F_{o}$ are the background and foreground (object) of the ground truth image, $B_{T}$ and $\mathrm{F}_{\mathrm{T}}$ the background and foreground pixels in the thresholded image and $|\cdot|$ is the cardinality of a set. The value of $M E$ varies between 0 for a perfectly classified image and 1 for a totally erroneously classified one. A lower value of $M E$ means better quality of corresponding thresholded image.

Table 1 Comparison is shown on the basis of Misclassification Error (ME) for proposed method

\begin{tabular}{|c|c|c|c|c|c|}
\hline Image name with size & Fixed window & $\begin{array}{l}\text { Wallner's } \\
\text { approach }\end{array}$ & $\begin{array}{c}\text { Derek bradley's } \\
\text { method }\end{array}$ & $\begin{array}{c}\text { Faisal shafait's } \\
\text { method }\end{array}$ & $\begin{array}{c}\text { Proposed } \\
\text { method }\end{array}$ \\
\hline Airplane $(321 \times 481)$ & 0.2366 & 0.1690 & 0.1254 & 0.1756 & 0.0806 \\
\hline $\operatorname{Bird}(256 \times 256)$ & 0.2811 & 0.1359 & 0.1307 & 0.2347 & 0.0362 \\
\hline Crow $(480 \times 320)$ & 0.1574 & 0.1398 & 0.1124 & 0.1559 & 0.0821 \\
\hline Elephant $(321 \times 481)$ & 0.3705 & 0.1814 & 0.1188 & 0.2748 & 0.0834 \\
\hline Flybird(480×320) & 0.0603 & 0.1218 & 0.0351 & 0.0798 & 0.0672 \\
\hline
\end{tabular}


Flow Chart

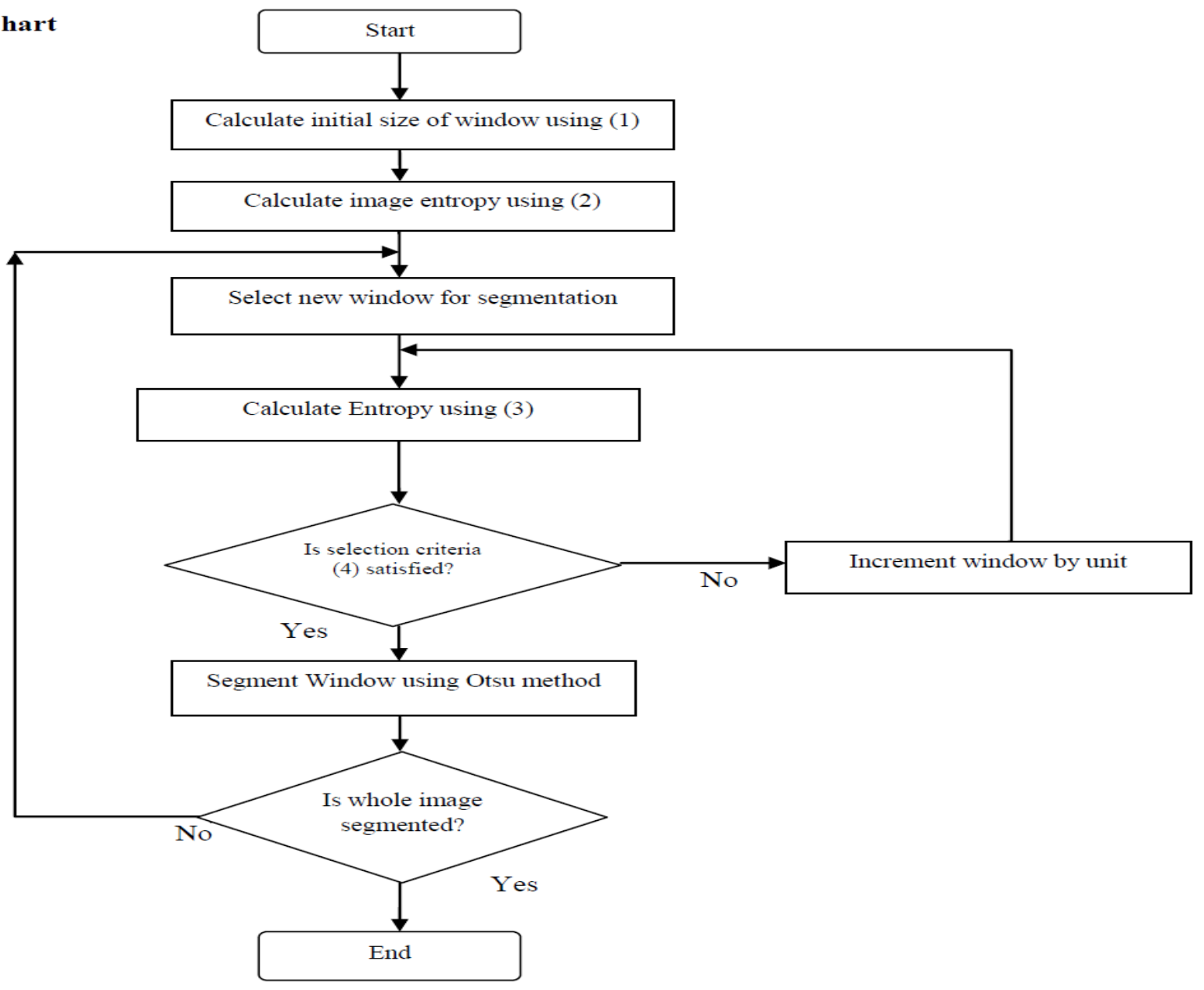

Fig. 1 Flow chart for proposed method

\section{RESULT AND ANALYSIS}

We have considered five gray level and compressed (jpeg) images $(($ airplane $(320 \times 480)$, bird $(256 \times 256)$, crow $(320 \times 480)$, elephant $(320 \times 480)$, flybird $(480 \times 320))$ of different dimension with reasonable non uniform illumination condition that are shown in Fig.2(a) to Fig.6(a). The corresponding ground truth images have been generated manually and are shown in Fig.2(c) to Fig.6(c). All the experiments are performed in MATLAB 7.0 using Core 2 Duo processor. To demonstrate the performance of the proposed method, segmentation result obtained by using proposed method is compared with some existed methods on the basis of percentage of Misclassification Error $(M E)$, comparison is shown in table 1. Segmentation result of the proposed method is compared with the Wallner's method[9], Darek bradley method's [6] and Faisal shafait's method[15]. In fixed window method, threshold is calculated by using same algorithm that is used to compute threshold value in the proposed method. In this method window size $(32 \times 32)$ is considered for all the images. It can be visualized from segmentation result that the proposed approach gives better performance than the fixed window approach for all the images. Wallner's[9] method compute pixel wise threshold, based on the scan line approach. Although this method is fast but produces $M E$ value (0.1218) minimum for the flybird image. Darek bradley[6] method makes use of integral image and required two pass in order to produce the segmentation result. The method is slower as compared to wallner method[9] but produce better segmentation result and also provide minimum $M E$ value for flybird image. Faisal's method[15] also use the integral image and compute threshold value using sauvola's method[8]. This method makes computation time independent of window size. In the proposed method, the selection criterion is based on the entropy value, and value of $C$ varies between $0-1$, for all the experiment value of $C$ is selected 0.40 . Proposed method partitions the image into dynamic size window thereafter segment the window. This method compute the initial size for images airplane, bird, crow elephant, flybird are $(22 \times 22)$, $(14 \times 14),(32 \times 32)(27 \times 27),(15 \times 15)$ respectively. It can be visualize from experimental result that proposed method give better result as compared to the method discussed above. 


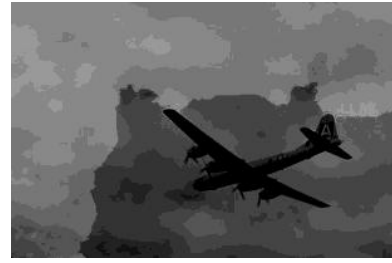

(a)

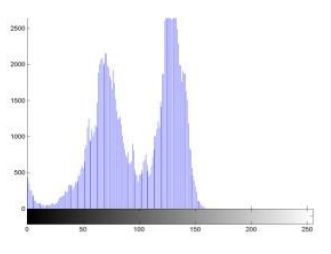

(b)

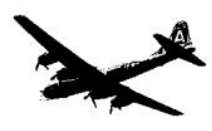

(c)

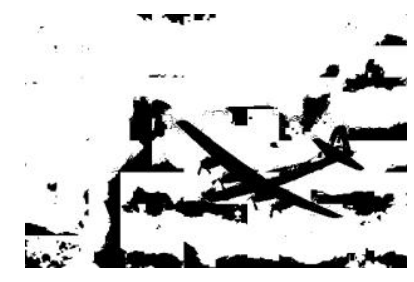

(d)

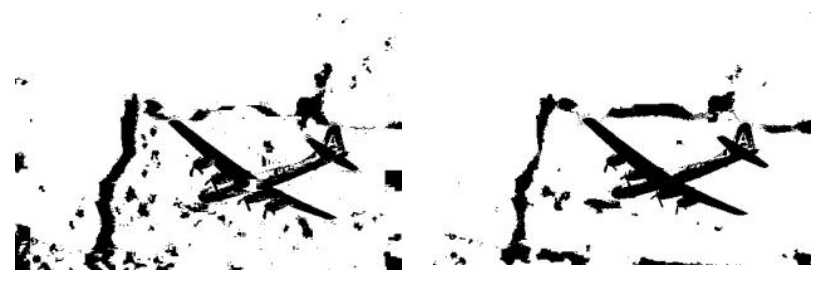

(e) (f)

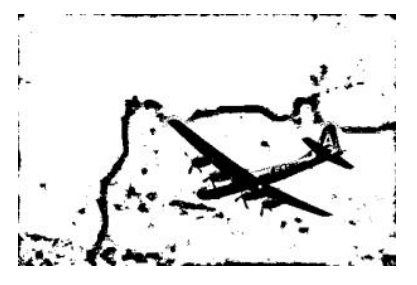

(g)

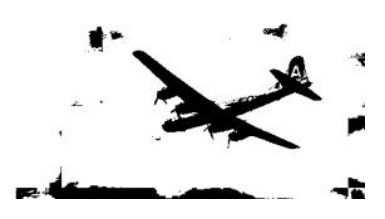

(h)

Fig. 2 (a) Original image of Airplane size $(321 \times 481)$ (b) Image histogram (c) Ground Truth image (d) Fixed window approach (e) Wallner's method (f) Derek bradley's method (g) Faisal shafait's method (h) Proposed method

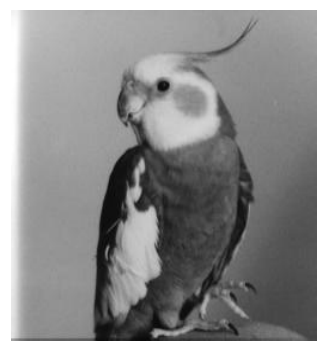

(a)

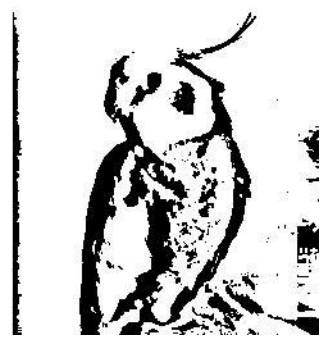

(e)

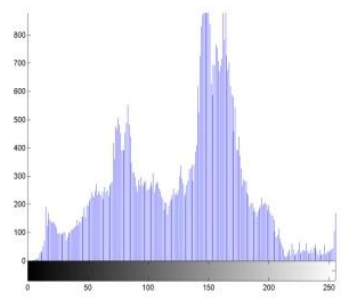

(b)

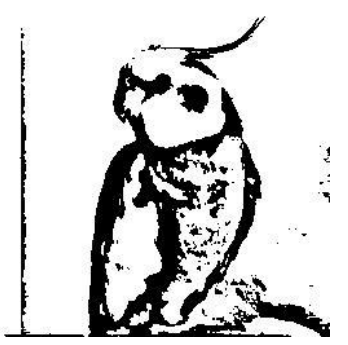

(f)

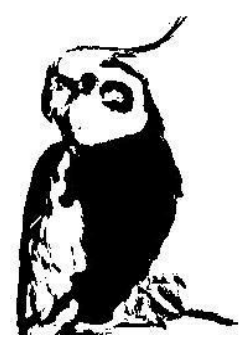

(c)

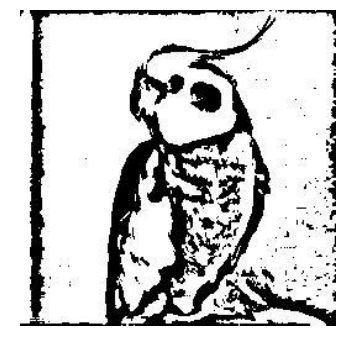

(g)

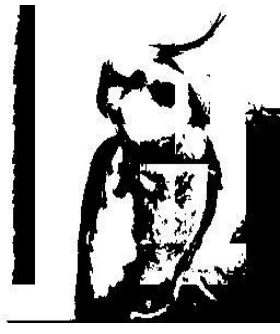

(d)

Fig. 3 (a) Original image of Bird size $(256 \times 256)$ (b)Image histogram (c) Ground Truth image (d) Fixed window approach (e) Wallner's method (f) Derek bradley's method (g) Faisal shafait's method (h) Proposed method

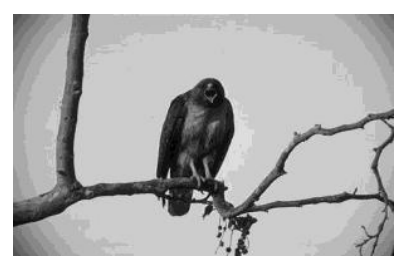

(a)

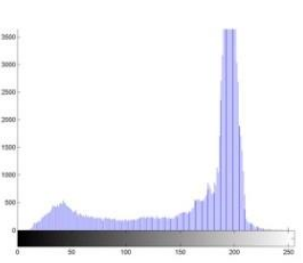

(b)

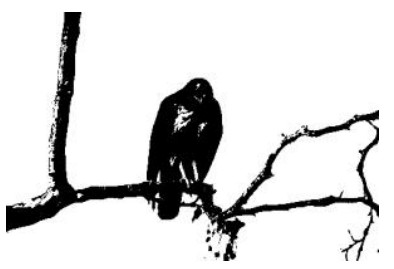

(c)

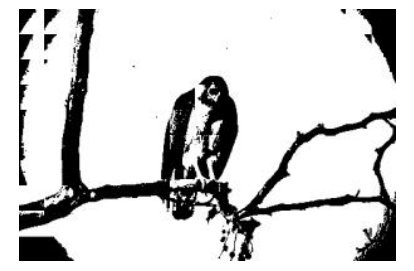

(d) 


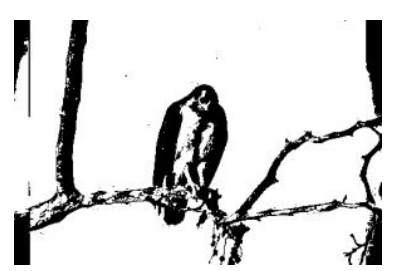

(e)

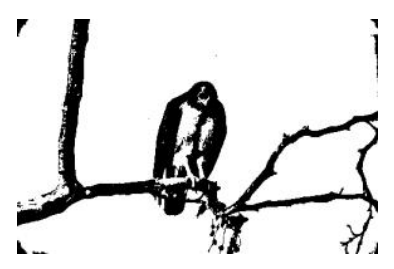

(f)

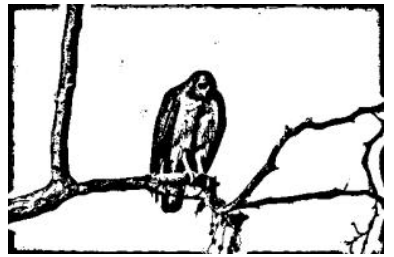

(g)

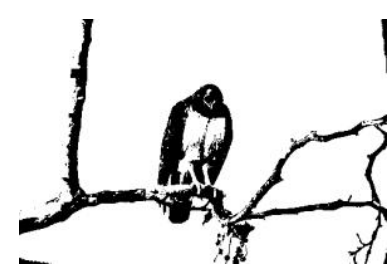

(h)

Fig. 4 (a) Original image of Crow size(320×480) (b) Image histogram (c) Ground Truth image (d) Fixed window approach (d) Wallner's method (e) Derek bradley's method (g) Faisal shafait's method (h) Proposed method

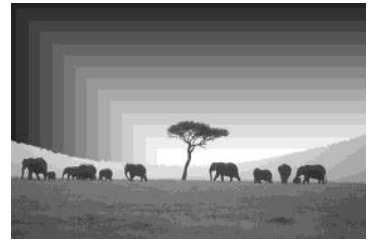

(a)

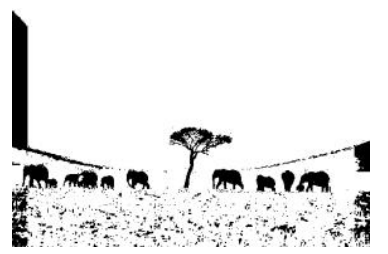

(e)

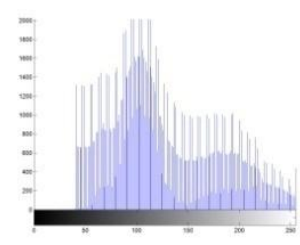

(b)

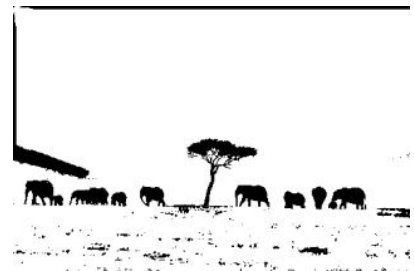

(f)

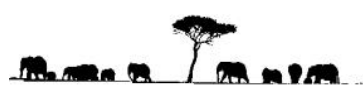

(c)

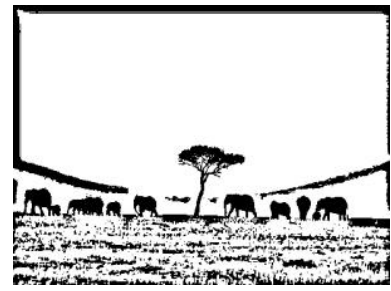

(g)

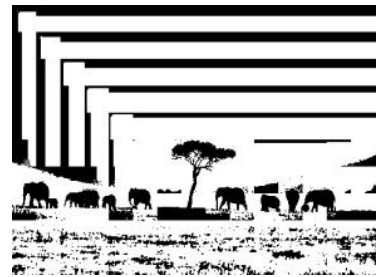

(d)

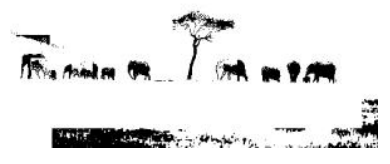

(h)

Fig.5 (a) Original image of Elephents size(321×481) (b) Image histogram (c)Ground Truth image (d) Fixed window method (e) Wallner's method (f)Derek bradley's method (g) Faisal shafait's method (h) Proposed method

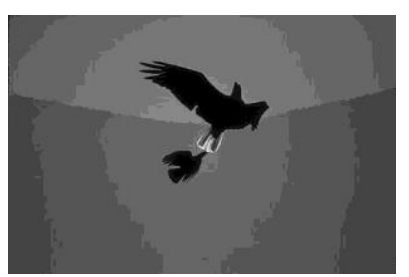

(a)

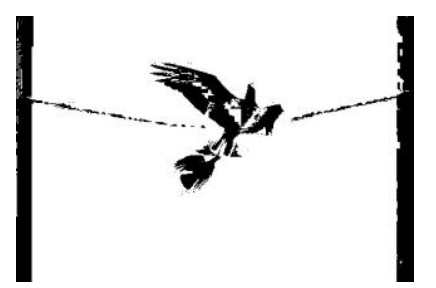

(e)

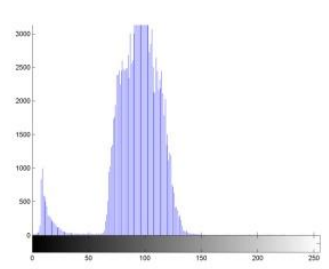

(b)

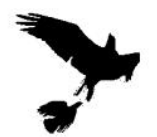

(c)

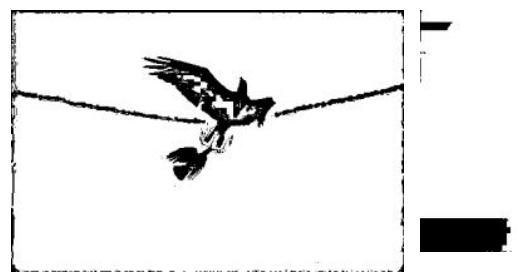

$(\mathrm{g})$

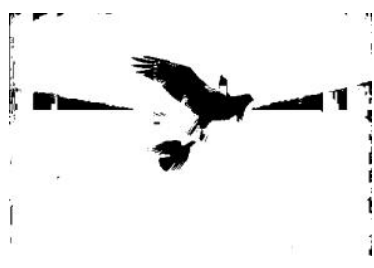

(d)

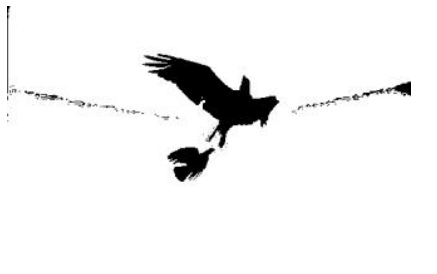

(f)

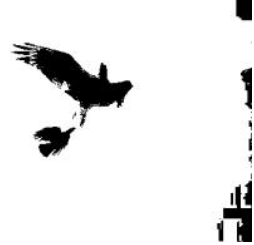

(h)

Fig. 6 (a) Original image of Flybird size $(321 \times 481)($ b) Image histogram (c)Ground Truth image (d) Fixed window method (e)Wallner's method (f) Derek bradley's method (g) Faisal shafait's method (h) Proposed method 


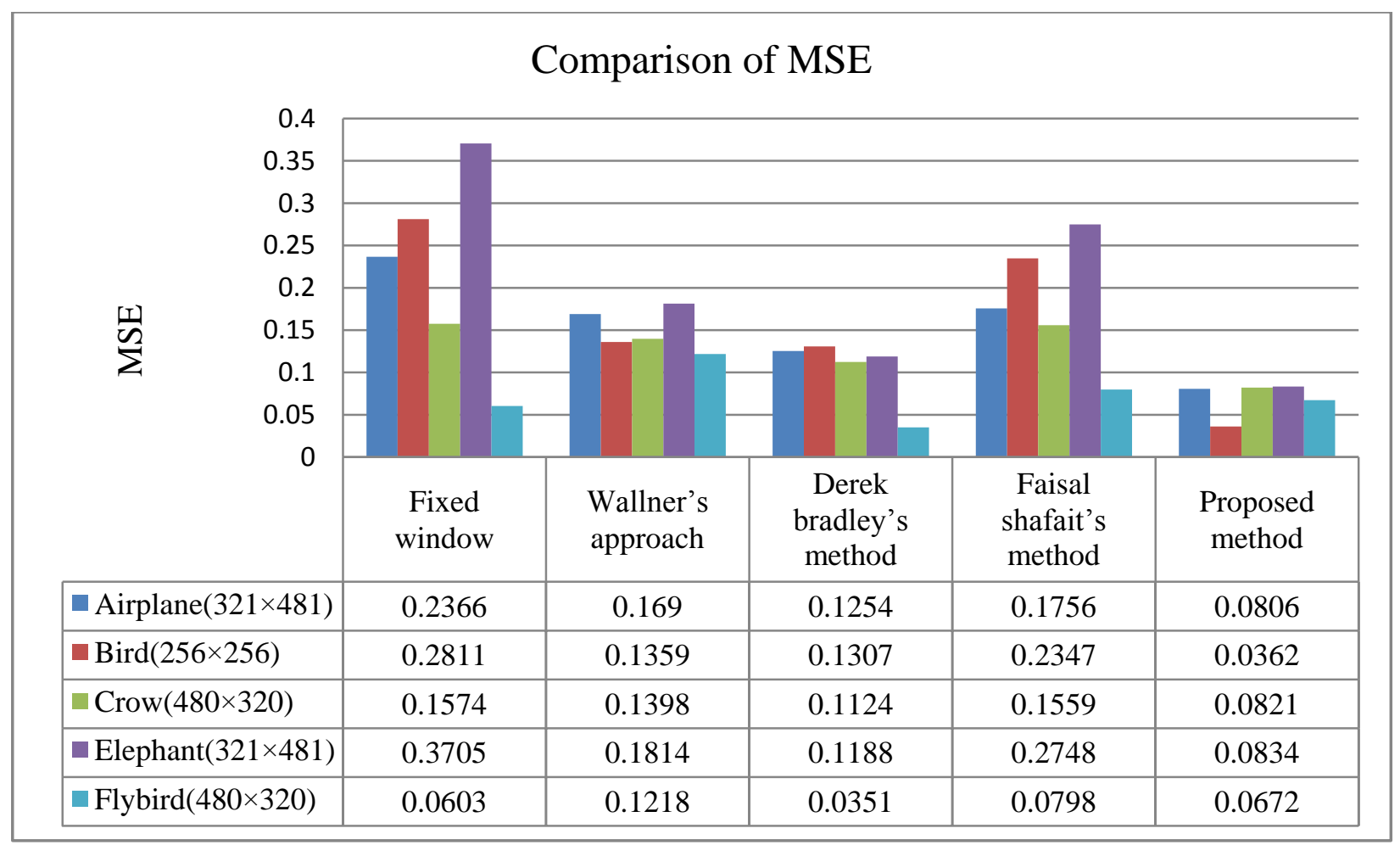

Fig. 7 Bar Chart of Misclassification Error of all images for all methods

\section{CONCLUSION}

In this paper, a novel method is presented for segmentation of images acquired under uneven lighting condition. Global thresholding approach failed to segment these images and hence adaptive thresholding methods are necessary. The proposed method is based on three steps: First compute the initial size of window using image statistics, a method to partition the images into dynamic size windows followed by the application of thresholding method on those windows. In window merging approach, it has been found that the segmentation accuracy greatly depends upon the proper choice of the initial window size. The selection of initial window size has been usually achieved by trial and error approach. In order to overcome this problem, initial window size is computed at runtime. In the proposed window growing approach, incremental value $\Delta \mathrm{w}$ is kept one. Evaluation is done on the basis of $M E$, comparison of proposed method with some existed effective method is shown in table1, and corresponding bar chart of $M E$ values is shown in Fig.7. The experimental result demonstrates the effectiveness of the proposed method.

\section{REFERENCES}

[1] N. Otsu, "A threshold selection method from gray-level histograms", IEEE Transaction on Systems Man and Cybernetics (SMC), Vol. 9, pp. 62-66, 1979

[2] Q. Huang, W. Gao, W. Cai, "Thresholding technique with adaptive window selection for uneven lighting image", Pattern Recognition Letters, Vol. 26, pp. 801808, 2005.

[3] Graham Leedham, Chen Yan, Kalyan Takru, Joie Hadi Nata Tan and Li Mian, "Comparison of Some Thresholding Algorithms for Text/Background Segmentation in Difficult Document Images", IEEE International conference on Document Analysis and Recognition (ICDAR), pp.859-864, 2003.
[4] Zuoyong Li, Yong Cheng, Chuancai Liu, Cairong Zhao, "Minimum Standard Deviation Difference-Based Thresholding", IEEE International Conference on Measuring Technology and Mechatronics Automation (ICMTMA), Vol. 2, pp. 664-667, 2010.

[5] P. Kanungo, P. K. Nanda, A. Ghosh, "Parallel Genetic Algorithm based adaptive thresholding for image segmentation under uneven lighting conditions" IEEE International Conference on System Man and Cybernetics (ICSMC), pp. 1904-1911, 2010.

[6] D. Bradley and Roth , "Adaptive thresholding using Integral image" Journal of graphics tool Vol. 12(2), pp. 13-21, 2007.

[7] Satyabrat Srikumar, Mamta Wagh, P.K. Nanda, "Adaptive Windowing and Granular Computing based Image Segmentation”, IEEE International Conference on Energy Automation and Signal (ICEAS), pp. 1-5, 2011

[8] J. Sauvola, M. Pietikainen, “Adaptive Document Image Binariation”, Pattern Recognition, Vol. 33, pp. 225- 236, 2000.

[9] Pierre D. Wellner "Adaptive thresholding for digital desk” Technical Report( EPC) pp. 93-110, 1993

[10] Bilal Bataineh, Siti Norul Huda, Sheikh Abdullah, Khairuddin Omar, "An adaptive local binarization method for document images based on a novel thresholding method and dynamic windows", Pattern Recognition Letters Vol. 32, pp. 1805-1813, 2011.

[11] Mehmet Sezgin, B. Sankur, "Survey over image thresholding techniques and quantitative performance evaluation", International Journal of Electronic Imaging(IJEI) Vol. 13 No. 1, pp.146-165, 2004.

[12] W. Niblack, "An Introduction to Digital Image Processing”, Prentice Hall, Englewood Cliffs, 1986. 
[13] Nikhil R.Pal and Sankar K.Pal, "A review on Image segmentation techniques", Pattern Recognition Vol. 26 No. 9 pp. 1277-1294, 1993.

[14] Feixiang Yan, Hong Zhang, C. Ronald Kube "A multistage adaptive thresholding method" Pattern Recognition Letters 26 , pp. 1183-1191, 2005.

[15] Faisal Shafait, Daniel Keysersa, Thomas M. Breuel, "Efficient Implementation of Local Adaptive Thresholding Techniques Using Integral Images", Proceedings of the International Society for Optical Engineering(SPIE), Vol. 68, pp. 510-516, 2008.

[16] Sahoo, P.K., Soltani, S., Wong, “ SURVEY: A survey of thresholding techniques" ,Computer Vision Graphics and Imahe Processing vol. 41, pp.233-260,1988.
[17] Spann, M., Wilson, R. “ A quad tree approach to image segmentation which combines statistical and spatial information" Pattern Recognition 18, pp. 257-269, 1985.

[18] Tsai, W.H.," Moment -preserving thresholding : A new approach,"Computer Vision Graphics and Imahe Processing vol.29, pp.377-393, 1985.

[19] Naveed Bin Rais, M. Shehzad Hanif and rmtiaz A. Taj, "Adaptive Thresholding Technique for Document Image Analysis", $8^{\text {th }}$ IEEE International Multitopic Conference, Vol. 3, pp. 61-66, 2004.

[20] W. A. Yasnoff, J. K. Mui, and J. W. Bacus, "Error measures for scene segmentation," Pattern Recognition, vol. 9(4), pp.217-231, 1977 